\title{
The Positive Modulation Effect of a 6-Week Consumption of an Anthocyanin-Rich Mulberry Milk on Working Memory, Cholinergic, and Monoaminergic Functions in Healthy Working- Age Adults
}

\author{
Wipawee Thukham-mee, ${ }^{1,2}$ Jintanaporn Wattanathorn (iD, ${ }^{1,2}$ Pongsatorn Paholpak, \\ Poonsri Ransikachi, ${ }^{1,3}$ and Nawanant Piyavhatkul ${ }^{1,3}$ \\ ${ }^{1}$ Research Institute for High Human Performance and Heath Promotion, Khon Kaen University, Khon Kaen 40000, Thailand \\ ${ }^{2}$ Department of Physiology, Faculty of Medicine, Khon Kaen University, Khon Kaen 40000, Thailand \\ ${ }^{3}$ Department of Psychiatry, Faculty of Medicine, Khon Kaen University, Khon Kaen 40000, Thailand
}

Correspondence should be addressed to Jintanaporn Wattanathorn; jinwat05@gmail.com

Received 21 January 2021; Accepted 10 August 2021; Published 2 September 2021

Academic Editor: Grzegorz Węgrzyn

Copyright (C) 2021 Wipawee Thukham-mee et al. This is an open access article distributed under the Creative Commons Attribution License, which permits unrestricted use, distribution, and reproduction in any medium, provided the original work is properly cited.

\begin{abstract}
Due to the increase of stress-related memory impairment accompanying with the COVID-19 pandemic and financial crisis, the prevention of cognitive decline induced by stress has gained much attention. Based on the evidence that an anthocyanin-rich mulberry milk demonstrated the cognitive enhancing effect, we hypothesized that it should be able to enhance memory in working-age volunteers who are exposed to working stress. This study is an open-label, two-arm randomized study. Both men and women volunteers at age between 18 and 60 years old were randomly assigned to consume the tested product either 1 or 2 servings daily for 6 weeks. All subjects were assessed for cortisol, acetylcholinesterase (AChE), monoamine oxidase (MAO), monoamine oxidase type A (MAO-A), and monoamine oxidase type B (MAO-B) in saliva, and their working memory was determined both at baseline and at a 6-week period. The results showed that the working memory of subjects in both groups was enhanced at the end of the study period together with the reduction of saliva cortisol. The suppression of AChE, MAO, and MAO-A was also observed in subjects who consumed the tested product 2 servings daily. Therefore, we suggest the memory enhancing effect of an anthocyanin-rich mulberry milk. The possible mechanism may occur primarily via the suppression of cortisol. In addition, the high dose of mulberry milk also suppresses AChE, MAO, and MAO-A.
\end{abstract}

\section{Introduction}

Stress, an inevitable event nowadays, is continually rising worldwide. Living in the era of the COVID-19 pandemic and financial crisis gives rise to the unintended adverse effects of stress including the increase risk of stress-related memory impairment $[1,2]$. Stress activates the hypothalamicpituitary-adrenocortical (HPA) axis which in turn gives rise to the release of glucocorticoids especially cortisol or stress hormone and results in memory deficit [3]. Due to the important roles of working memory on a daily life activity and on career success together with the increasing stress prevalence, the pre- vention of stress-related cognitive impairment has gained much attention.

Mulberry or Morus alba, a plant in the family of Moraceae, has been widely planted in Thailand. The ripe fruit of mulberry has been consumed both as a fruit and as medicine. Our previous studies have demonstrated that it possesses antioxidant, anti-inflammation neuroprotection, and memory enhancer [4-7]. Recently, we have demonstrated the clinical evidence that within 1.5 hours after the consumption of an anthocyanin-rich mulberry milk which contains mulberry fruit $10 \mathrm{~g}$, the children at the age between 6 and 12 years old show the reduction of saliva cortisol together with the memory enhancement [8]. 
Based on this information, we hypothesized that a 6-week consumption of an anthocyanins-rich mulberry milk should be able to decrease stress hormone and improve working memory in healthy working-age volunteers. Due to the lack of available data concerning this point, this study is set up to test this hypothesis.

\section{Materials and Methods}

2.1. Anthocyanin-Rich Mulberry Milk. Anthocyanin-rich mulberry milk (Memberry ${ }^{\circledR}$ ) used in this study was kindly supported by Mark One Innovation Center Company Limited of MK Restaurant Group. Each serving $(180 \mathrm{ml})$ contains total phenolic compounds, flavonoids, and anthocyanins at the concentrations of $1415.17 \pm 1.85 / \mathrm{mg} \mu \mathrm{g}$ GAE, $667 \pm 1.07 \mu \mathrm{g}$ quercetin/mg, and $34.30 \pm 0.74 \mathrm{mg}$ cyanidin glucoside equivalent/L, respectively.

2.2. Study Design. This open-label, two-arm randomized study was designed to collect data on the potential effect of anthocyanin-rich mulberry milk consumption for 6 weeks on working memory in healthy working-age adults. The possible underlying mechanisms were also explored. It was performed according to the Declaration of Helsinki (ethical principles for research involving human subjects), and all procedures were under the approval of the Institute Human Ethical Committee under HE631051. This project was also registered online as part of the Thai Clinical Trial Registry (TCTR20201031002).

2.3. Participants and Interventions. A total of healthy 312 men and women at the age between 18 and 60 years old were recruited, and they were screened for eligibility. Only 300 subjects participated in this study. Individuals receiving treatment with any medications or herbs that may exert the influence on brain function or who had any prior diagnosis or history of stroke, heart disease, diabetes, gastrointestinal disease, cancer, central nervous system or psychiatric disorders, or traumatic brain injury were excluded. In addition, heavy smokers and alcohol addicts were also excluded. All participants provided written informed consent before participating in the study. Recruited subjects were randomly assigned to consume either one $(180 \mathrm{ml})$ or two servings $(360 \mathrm{ml})$ of mulberry milk daily for 6 weeks. All subjects still maintain their habitual diet and physical activity levels during the study period. They were given customized calendars and were asked to mark the days they missed consuming the assigned product. Unused study products were returned for compliance monitoring purposes.

Both primary and secondary outcomes of the subjects at baseline or before the intervention and at 6 weeks after the intervention were measured. The primary outcome in this study was working memory assessed by the computerized battery test consisting of word presentation, word recognition test, picture presentation, picture recognition, simple reaction time, digit vigilance, choice reaction time, and spatial working memory whereas the secondary outcomes were salivary cortisol, acetylcholinesterase, monoamine oxidase (MAO), monoamine oxidase type $\mathrm{A}\left(\mathrm{MAO}_{\mathrm{A}}\right)$, and type
$\mathrm{B}\left(\mathrm{MAO}_{\mathrm{B}}\right)$. The schematic diagram illustrating the subject intervention procedure is shown in Figure 1.

2.4. Computerized Battery Test. The computerized battery test used in this study measured 4 domains of working memory comprising attention, continuity of attention, quality of memory, and speed of memory [9-11]. The test-retest reliability in each domain of working memory varied between 0.72 and 0.82 (power of attention $=0.75$, continuity of attention $=0.78$, quality of working memory $=0.82$, and speed of memory $=0.72$ ). The presentation of each task was performed via VGA color monitors. The responses of all tasks were recorded via a yes or no button box. The entire battery tests were around 20 minutes. The sequence of the test was performed as described below:

Word presentation: a subject was exposed with a 15word presentation which was matched for frequency and concreteness. The stimulus duration was $1 \mathrm{~s}$, as was the interstimulus interval. Both response and percentage of response accuracy were recorded.

Picture presentation: according to this test, each subject was exposed with a 20 photographic image exposure. The presentation was performed on the monitor at the rate of 1 every $3 \mathrm{~s}$, with a stimulus duration of $1 \mathrm{~s}$, for the participant to remember.

Simple reaction time: in this test, a 15-stimulus presentation was shown with an interstimulus interval that randomly varied between 1 and $3.5 \mathrm{~s}$. All subjects were instructed to press the "yes" response button as quickly as possible every time the word "yes" was presented on the monitor. All response reaction times were recorded in milliseconds.

Digit vigilance task: each subject was exposed to 15 stimuli of digit presentation. The target digit was randomly selected and shown on the right side of the monitor screen, whereas the series of digit presentation was performed at the center of the screen at the rate of $80 \mathrm{~min}^{-1}$. All subjects were instructed to the "yes" button as quickly as possible every time the digit in the series matched the target digit.

Choice reaction time: all subjects were subjected to either the word "no" or the word "yes." They were instructed to press the corresponding button as quickly as possible when the word presentation appeared on the monitor. Each set consisted of 50 trials. The stimulus word was randomly chosen with equal probability, with the interstimulus interval between 1 and 3.5 seconds.

Spatial working memory: according to this test, each subject was exposed to a targeted house picture with 9 windows. Four windows in this picture were illuminated. The subjects were instructed to memorize the position of the illuminated windows in the targeted house picture. Then, they were exposed to a series of house presentations with one illuminated window. The subject must match whether the illuminated window of each presented picture was shown at the location of the targeted house picture by pressing the "yes" or "no" response button as quickly as possible.

Numeric working memory: the subjects were exposed to 5 digits sequentially and they were instructed to memorize them. Following this process, a series of 30 probe digits were presented to the subjects and they must decide whether the 


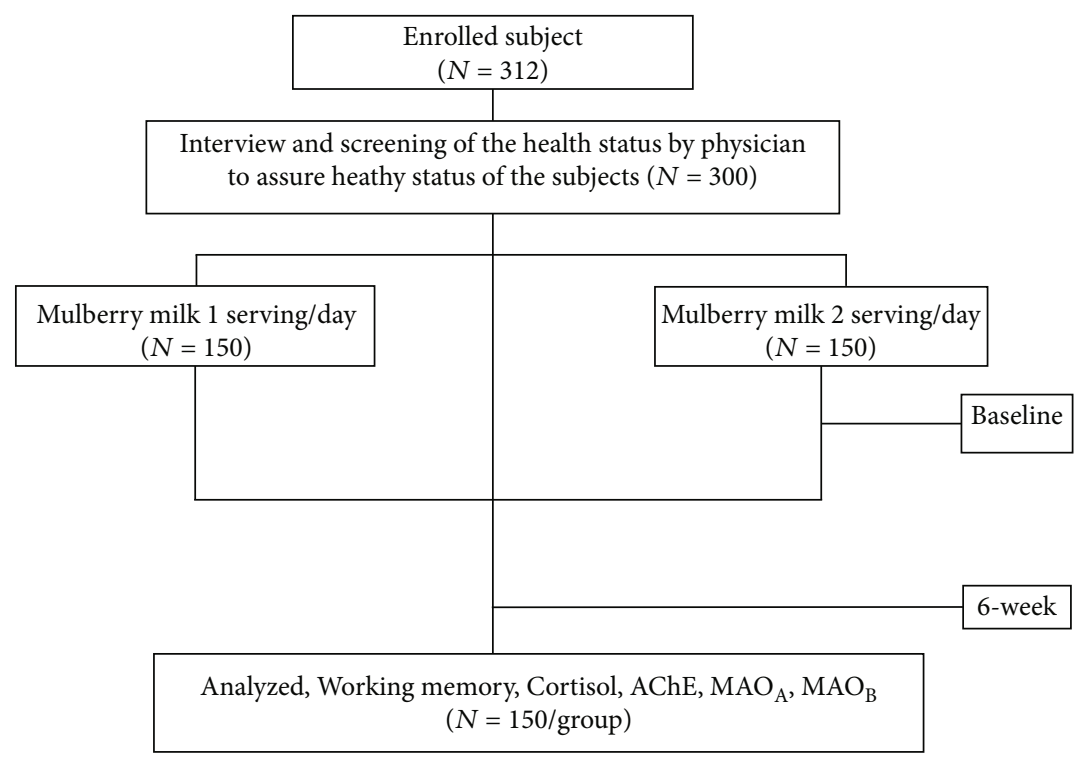

FIGURE 1: Flow diagram of subject distribution and study design.

probe digit was matched with the digits which previously exposed and must press the "yes" or "no" response button as quickly as possible.

\subsection{Biochemical Assays}

2.5.1. Saliva Collection. Saliva samples were collected by using a Salivette collection device kit (No. 51.1534; Sarstedt, Numbrecht, Germany), according to the guideline. In brief, the sample was collected between 7.00 and 8.00 a.m. before breakfast. Each subject must rest for $1 \mathrm{~min}$ in a sitting position before saliva collection. Swab was directly placed into the subject's mouth under the tongue or allowed to move freely across the tongue. Do not place the swab between the cheek and gum and keep the swab in the mouth for two to three minutes to assure that the swab was completely saturated with enough volume (1/4 teaspoon was required). The samples were immediately frozen and transported to the laboratory for assays.

2.5.2. Saliva Cortisol Assessment. The assessment of saliva cortisol was carried out by using ELISA kit. Briefly, $25 \mu \mathrm{l}$ of standard or saliva was mixed with $200 \mu \mathrm{l}$ of cortisol-HRP conjugate solution and incubated $37^{\circ} \mathrm{C}$ for 60 minutes. At the end of the incubation period, the solution was washed with buffer for three times and TMB (tetramethylbenzidine) substrate solution at the volume of $100 \mu \mathrm{l}$ was added and incubated at the room temperature for 15 minutes. Following this process, the stop solution was added. An absorbance at $450 \mathrm{~nm}$ was recorded via a microplate reader by using cortisol as a standard reference. The results were expressed as nanograms/milliliter.

2.5.3. Determination of Acetylcholinesterase (AChE). Saliva acetylcholinesterase (AChE) has been recognized as a valid indicator to indicate central cholinergic activity [12] so a saliva AChE was measured to explore the effect of anthocyanin-rich mulberry milk on the central cholinergic system. In brief, an aliquot of saliva or control at the volume of $10 \mu \mathrm{l}$ was mixed with the solution containing $10 \mu \mathrm{l}$ of $0.2 \mathrm{mM}$ DTNB, $20 \mu \mathrm{l}$ of $0.1 \mathrm{mM}$ PBS pH 8.0, and $10 \mu \mathrm{l}$ of $15 \mathrm{mM}$ ATCI in the 96-well plates and subjected to a 5 minute-incubation period at room temperature. Following this step, an absorbance at $415 \mathrm{~nm}$ was recorded. The calculation of AChE activity was performed according to the following equation:

$$
\text { AChE activity }=\left(\frac{\Delta A}{1.36 \times 10^{4}}\right) \times \frac{1}{(20 / 230) C},
$$

where $\Delta A$ is the difference of absorbance/minute and $C$ is protein concentration of brain homogenate.

2.6. Assessment of Monoamine Oxidase Enzyme Activity. Due to the correlation between saliva monoamine oxidase activity of MAO, MAO-A, and MAO-B and stress [13], the activities of the mentioned enzymes were also assessed in this study. The monoamine oxidase activity was determined by using the modified method of Holt and coworkers [14]. In brief, an aliquot of saliva at the volume of $50 \mu \mathrm{l}$ was mixed with a chromogenic solution consisting of $1 \mathrm{mM}$ vanillic acid, $500 \mu \mathrm{M} 4$-aminoantipyrine, and $4 \mathrm{U} \cdot \mathrm{ml}$ peroxidase in $0.2 \mathrm{M}$ potassium phosphate buffer, $\mathrm{pH}$ 7.6. After mixing thoroughly, tyramine which was used as substrate was added and incubated at $37^{\circ} \mathrm{C}$ for 30 minutes. At the end of an incubation period, the absorbance at $490 \mathrm{~nm}$ was recorded with a microplate reader (iMark ${ }^{\mathrm{TM}}$ Microplate Absorbance Reader). All data values were expressed as nanomoles/hour/milligram protein.

To measure the activity of MAO-A, each saliva sample at the volume of $50 \mu \mathrm{l}$ was transferred into a 96 -well plate containing $50 \mu \mathrm{l}$ of $500 \mathrm{nM}$ pargyline (MAO-A inhibitor) and subjected to a 30-minute incubation period at room temperature to allow the blocking of MAO-A activity by pargyline. Following this step, $200 \mu \mathrm{l}, 500 \mu \mathrm{M}$ tyramine was added to all wells. Then, the mixtures were incubated for 30 minutes 
at room temperature in the dark condition. According to this method, $p$-tyramine was used as substrate. At the end of incubation period, they were measured absorbance at the wavelength of $490 \mathrm{~nm}$.

MAO-B activity was also determined by the same procedure but clorgyline was used as a substrate. The preparation of standard was also carried out as mentioned above but $\mathrm{H}_{2} \mathrm{O}_{2}$ was used to replace the saliva sample.

2.7. Statistical Analysis. All parameters were expressed as mean \pm SEM. The statistical analysis was performed by using the Student paired $t$-test. The significance was considered when the $p$ value was less than 0.05 .

\section{Results}

3.1. Participant Flow and General Characteristics. A total of 300 healthy participants who met the study criteria from 312 enrolled subjects were randomly assigned to the 1 serving consumption (150) and 2 serving consumption (150) groups. No one dropped out from the study before the study completion as shown in Figure 1.

The general characteristics of subjects who consumed mulberry milk at dose of 1 serving and 2 serving per day are shown in Table 1. It was found that after 6 weeks of consumption, subjects who consumed mulberry milk 2 servings per day showed the significant elevation in pulse rate $(p$ value $<0.05)$ and respiratory rate $(p$ value $<0.01)$ whereas the subjects who consumed mulberry milk 1 serving per day showed the significant reduction in diastolic pressure ( $p$ value $<0.05$ ). No other significant changes of any parameters were observed.

3.2. Effect on Working Memory. The effects of mulberry milk on working memory of subjects who consumed mulberry milk at a dose of 1 and 2 servings daily are shown in Tables 2 and 3. After a 6-week consumption period of mulberry milk, the subjects who consumed mulberry milk 1 and 2 servings per day showed the significant reduction of response time in word recognition, picture recognition, simple reaction, choice reaction time, spatial memory, and numeric working memory ( $p$ value $<0.001$ all; $p$ value $<0.001$ and $0.01 ; p$ value $<0.001$ all; $p$ value $<0.05$ and $0.01 ; p$ value $<0.001$ all; and $p$ value $<0.001$ all). In addition, they also showed the significant increase in \%accuracy in word recognition, picture recognition, digit vigilance, and spatial time ( $p$ value $<0.001$ all; $p$ value $<0.001$ all; $p$ value $<0.05$ and 0.01 ; and $p$ value $<0.01$ all).

3.3. Biochemical Assays. Tables 4 and 5 showed the effect of mulberry milk on saliva cortisol and on the changes of salivary changes of AChE, MAO, MAO-A, and MAO-B. It was found that only saliva cortisol showed the significant reduction in subjects who consumed mulberry milk 1 serving per day ( $p$ value $<0.001$ ). However, subjects who consumed mulberry milk at a dose of 2 servings per day significantly showed to have decreased cortisol, AChE, MAO, and MAO-A in saliva ( $p$ value $<0.001,0.05,0.05$, and 0.05 , respectively).

\section{Discussion}

In the study of cognitive function, especially in learning and memory area, one capability that gains much attention is the assessment of power of attention, which includes the abilities of attention and information processing [15]. This capability can be assessed by using response times in simple reaction time, choice reaction time, and digit vigilance task forces as indices because the response time reflects how the subjects pay attention to the stimuli. The more shorter response time indicates the better attention toward stimuli. Therefore, the present results indicate the increase in power of attention induced by anthocyanin-rich mulberry milk at both doses used in this study. In addition, the continuity of attention or the ability to maintain attention is also focused. It has been demonstrated that anthocyanin-rich mulberry milk also increases this ability manifested by the increase in percent accuracy in the performances of simple reaction time, choice reaction time, and digit vigilance tests. The computerized battery test can assess not only attention but also memory. Our data also reveal that subjects who consume anthocyanin-rich mulberry milk both at 1 serving and 2 servings per day significantly improve both response time and percent accuracy in word recognition, picture recognition, spatial, and numeric working memory. These changes indicate the improvement of quality and speed of memory. Therefore, our data reveal that anthocyanin-rich mulberry milk can improve 4 domains of working memory including attention, continuity of attention, speed of memory, and quality of memory.

Recently, it has been demonstrated that working memory shows a negative relationship with cortisol and AChE [8]. Therefore, we also investigate the effect of anthocyanin-rich mulberry milk on the mentioned parameters after 6 weeks of consumption period. Our data also confirm the effect of mulberry milk. It has been revealed that the saliva cortisol shows a significant decrease after a 6-week consumption period of anthocyanin-rich mulberry milk 1 serving per day. No change of AChE is observed. The significant reduction of both saliva cortisol and AChE is observed in subjects who consume an anthocyanin-rich mulberry milk 2 servings per day. In addition to the changes of the mentioned parameters, the significant reduction of MAO and MAO-A is also observed. It has been previously shown that the MAO and MAO-A are associated with working memory [16-20]. The suppression of MAO activity is reported to produce the improvement of memory impairment [20]. Due to these pieces of information, we do suggest that the enhancement of memory induced by an anthocyanin-rich mulberry milk may occur partly via the suppression of cortisol, AChE, MAO, and MAO-A. Based on the suppression effect of anthocyanin on AChE [21], MAO, and MAO-A [22], we suggest that the possible active ingredient may partly involve anthocyanin content in the mulberry milk.

Our data also demonstrate that subjects who consumed an anthocyanin-rich mulberry milk 2 servings daily also show the elevation of pulse rate, which indicates the elevation of heart rate, which in turn should enhance blood pressure. However, no significant change in blood pressure is observed. The possible underlying mechanism may involve 
TABLE 1: The characterization of subjects. Values are presented as mean \pm SEM ( $n=150$ /group). Comparison of data was performed between at baseline and at a 6 -week study period. ${ }^{*} p$ value $<0.05,{ }^{* *} p$ value $<0.01$, respectively.

\begin{tabular}{|c|c|c|c|c|c|c|}
\hline \multirow{2}{*}{ Characteristics } & \multicolumn{2}{|c|}{ Mulberry milk 1 serving/day } & \multirow{2}{*}{$p$ value } & \multicolumn{2}{|c|}{ Mulberry milk 2 servings/day } & \multirow{2}{*}{$p$ value } \\
\hline & Baseline & 6 weeks & & Baseline & 6 weeks & \\
\hline Age (year) & $36.39 \pm 0.63$ & $36.39 \pm 0.63$ & 1 & $34.84 \pm 0.65$ & $34.84 \pm 0.65$ & 1 \\
\hline Gender (male/female) & $44 / 106$ & $44 / 106$ & & $38 / 112$ & $38 / 112$ & \\
\hline PR (beats/min) & $78.05 \pm 0.90$ & $79.78 \pm 0.85$ & 0.162 & $78.53 \pm 0.97$ & $81.34 \pm 0.97$ & $0.041^{*}$ \\
\hline Respiratory rate (breaths/min) & $17.50 \pm 0.17$ & $17.73 \pm 0.12$ & 0.256 & $17.09 \pm 0.17$ & $17.65 \pm 0.13$ & $0.009^{* *}$ \\
\hline Systolic BP (mmHg) & $123.43 \pm 1.08$ & $122.37 \pm 1.04$ & 0.482 & $123.01 \pm 1.14$ & $122.01 \pm 1.09$ & 0.525 \\
\hline Diastolic BP (mmHg) & $80.62 \pm 0.80$ & $78.05 \pm 0.76$ & $0.021^{*}$ & $80.37 \pm 0.83$ & $78.59 \pm 0.90$ & 0.148 \\
\hline Body weight (kg) & $61.63 \pm 0.95$ & $61.82 \pm 0.96$ & 0.893 & $63.28 \pm 1.02$ & $63.47 \pm 1.00$ & 0.895 \\
\hline Body height $(\mathrm{cm})$ & $160.70 \pm 0.59$ & $160.69 \pm 0.59$ & 0.987 & $161.12 \pm 0.60$ & $161.14 \pm 0.60$ & 0.988 \\
\hline Body mass index $\left(\mathrm{kg} / \mathrm{m}^{2}\right)$ & $23.80 \pm 0.31$ & $23.87 \pm 0.31$ & 0.876 & $24.41 \pm 0.39$ & $24.48 \pm 0.39$ & 0.891 \\
\hline
\end{tabular}

TABLE 2: Effect of mulberry milk consumption 1 serving per day on percent of accuracy response and response time of each cognitive assessment test. Values are presented as mean \pm SEM $(n=150$ /group). Comparison of data was performed between at baseline and at a 6-week study period. ${ }^{*} p$ value $<0.05,{ }^{* *} p$ value $<0.01$, and ${ }^{* * *} p$ value $<0.01$, respectively.

\begin{tabular}{|c|c|c|c|c|}
\hline Cognitive domains & Test items & \multicolumn{2}{|c|}{ Mulberry milk 1 serving/day } & $p$ value \\
\hline \multirow{4}{*}{ Word recognition } & \multirow{2}{*}{ Response time } & Baseline & $1304.81 \pm 22.40$ & \multirow{2}{*}{$p<0.001$} \\
\hline & & 6 weeks & $1136.83 \pm 15.06^{* * *}$ & \\
\hline & \multirow{2}{*}{ \%accuracy } & Baseline & $84.12 \pm 0.78$ & \multirow{2}{*}{$p<0.001$} \\
\hline & & 6 weeks & $87.77 \pm 0.66^{* * *}$ & \\
\hline \multirow{4}{*}{ Picture recognition } & \multirow{2}{*}{ Response time } & Baseline & $133.93 \pm 24.56$ & \multirow{2}{*}{$p<0.001$} \\
\hline & & 6 weeks & $1225.47 \pm 18.06^{* * *}$ & \\
\hline & \multirow{2}{*}{ \%accuracy } & Baseline & $86.68 \pm 0.62$ & \multirow{2}{*}{$p<0.001$} \\
\hline & & 6 weeks & $90.07 \pm 0.59^{* * *}$ & \\
\hline \multirow{2}{*}{ Simple reaction } & \multirow{2}{*}{ Response time } & Baseline & $650.95 \pm 13.97$ & \multirow{2}{*}{$p<0.001$} \\
\hline & & 6 weeks & $578.42 \pm 9.74^{* * *}$ & \\
\hline \multirow{4}{*}{ Digit vigilance } & \multirow{2}{*}{ Response time } & Baseline & $623.68 \pm 4.51$ & \multirow{2}{*}{$p=0.477$} \\
\hline & & 6 weeks & $619.33 \pm 4.13$ & \\
\hline & \multirow{2}{*}{ \%accuracy } & Baseline & $96.10 \pm 0.24$ & \multirow{2}{*}{$p=0.017$} \\
\hline & & 6 weeks & $69.98 \pm 0.27^{*}$ & \\
\hline \multirow{4}{*}{ Choice reaction time } & \multirow{2}{*}{ Response time } & Baseline & $776.97 \pm 0.21$ & \multirow{2}{*}{$p=0.010$} \\
\hline & & 6 weeks & $745.54 \pm 8.16^{*}$ & \\
\hline & \multirow{2}{*}{ \%accuracy } & Baseline & $97.90 \pm 0.21$ & \multirow{2}{*}{$p=0.534$} \\
\hline & & 6 weeks & $98.05 \pm 0.14$ & \\
\hline \multirow{4}{*}{ Spatial memory } & \multirow{2}{*}{ Response time } & Baseline & $1405.60 \pm 29.97$ & \multirow{2}{*}{$p<0.001$} \\
\hline & & 6 weeks & $1269.92 \pm 22.72^{* * *}$ & \\
\hline & \multirow{2}{*}{ \%accuracy } & Baseline & $90.76 \pm 0.97$ & \multirow{2}{*}{$p=0.002$} \\
\hline & & 6 weeks & $94.45 \pm 0.63^{* *}$ & \\
\hline \multirow{4}{*}{ Numeric working memory } & \multirow{2}{*}{ Response time } & Baseline & $11616.85 \pm 16.63$ & \multirow{2}{*}{$p=0.006$} \\
\hline & & 6 weeks & $1053.39 \pm 15.93^{* *}$ & \\
\hline & \multirow{2}{*}{ \%accuracy } & Baseline & $94.27 \pm 0.76$ & $n-0,017$ \\
\hline & & 6 weeks & $96.49 \pm 0.53^{*}$ & $p-0.01$ \\
\hline
\end{tabular}


TABLE 3: Effect of mulberry milk consumption 2 servings per day on percent of accuracy response and response time of each cognitive assessment test. Values are presented as mean \pm SEM ( $n=150$ /group). Comparison of data was performed between at baseline and at a 6-week study period. ${ }^{*} p$ value $<0.05,{ }^{* *} p$ value $<0.01$, and ${ }^{* * *} p$ value $<0.01$, respectively.

\begin{tabular}{|c|c|c|c|c|}
\hline Cognitive domains & Test items & \multicolumn{2}{|c|}{ Mulberry milk 2 servings/day } & $p$ value \\
\hline \multirow{4}{*}{ Word recognition } & \multirow{2}{*}{ Response time } & Baseline & $1335.46 \pm 21.12$ & \multirow{2}{*}{$p<0.001$} \\
\hline & & 6 weeks & $1143.61 \pm 0.62^{* * *}$ & \\
\hline & \multirow{2}{*}{ \%accuracy } & Baseline & $83.57 \pm 0.76$ & \multirow{2}{*}{$p<0.001$} \\
\hline & & 6 weeks & $88.15 \pm 0.62^{* * *}$ & \\
\hline \multirow{4}{*}{ Picture recognition } & \multirow{2}{*}{ Response time } & Baseline & $1336.74 \pm 26.72$ & \multirow{2}{*}{$p=0.006$} \\
\hline & & 6 weeks & $1246.17 \pm 18.61^{* *}$ & \\
\hline & \multirow{2}{*}{ \%accuracy } & Baseline & $87.28 \pm 0.68$ & \multirow{2}{*}{$p<0.001$} \\
\hline & & 6 weeks & $91.12 \pm 0.68^{* * *}$ & \\
\hline \multirow{2}{*}{ Simple reaction } & \multirow{2}{*}{ Response time } & Baseline & $666.34 \pm 17.29$ & \multirow{2}{*}{$p<0.001$} \\
\hline & & 6 weeks & $577.41 \pm 8.92^{* * *}$ & \\
\hline \multirow{4}{*}{ Digit vigilance } & \multirow{2}{*}{ Response time } & Baseline & $621.98 \pm 4.34$ & \multirow{2}{*}{$p=0.654$} \\
\hline & & 6 weeks & $619.31 \pm 4.08$ & \\
\hline & \multirow{2}{*}{ \%accuracy } & Baseline & $95.93 \pm 0.30$ & \multirow{2}{*}{$p=0.002$} \\
\hline & & 6 weeks & $97.11 \pm 0.23^{* *}$ & \\
\hline \multirow{4}{*}{ Choice reaction time } & \multirow{2}{*}{ Response time } & Baseline & $779.86 \pm 10.76$ & \multirow{2}{*}{$p=0.004$} \\
\hline & & 6 weeks & $741.20 \pm 7.88^{* *}$ & \\
\hline & \multirow{2}{*}{ \%accuracy } & Baseline & $97.74 \pm 0.18$ & \multirow{2}{*}{$p=0.300$} \\
\hline & & 6 weeks & $97.99 \pm 0.16$ & \\
\hline \multirow{4}{*}{ Spatial memory } & \multirow{2}{*}{ Response time } & Baseline & $1384.93 \pm 27.99$ & \multirow{2}{*}{$p<0.001$} \\
\hline & & 6 weeks & $1227.99 \pm 19.34^{* * *}$ & \\
\hline & \multirow{2}{*}{ \%accuracy } & Baseline & $91.43 \pm 1.09$ & \multirow{2}{*}{$p=0.007$} \\
\hline & & 6 weeks & $94.87 \pm 0.66^{* *}$ & \\
\hline \multirow{4}{*}{ Numeric working memory } & \multirow{2}{*}{ Response time } & Baseline & $1098.42 \pm 16.65$ & \multirow{2}{*}{$p=0.008$} \\
\hline & & 6 weeks & $1039.73 \pm 14.22^{* *}$ & \\
\hline & \multirow{2}{*}{ \%accuracy } & Baseline & $95.73 \pm 0.55$ & $D=0334$ \\
\hline & & 6 weeks & $96.45 \pm 0.51$ & $P-0.004$ \\
\hline
\end{tabular}

TABLE 4: The effect of mulberry milk 1 serving/day on cortisol level and the activities of AChE, $\mathrm{MAO}, \mathrm{MAO}_{\mathrm{A}}$, and $\mathrm{MAO}_{\mathrm{B}}$ in saliva $(n=150$ /group). ${ }^{* * *} p$ value $<0.001$ compared to baseline.

\begin{tabular}{|c|c|c|c|}
\hline \multirow{2}{*}{ Characteristics } & \multicolumn{2}{|c|}{ Mulberry milk 1 serving/day } & \multirow{2}{*}{$p$ value } \\
\hline & Baseline & 6 weeks & \\
\hline Cortisol level (ng/ml) & $8.40 \pm 0.12$ & $6.94 \pm 0.10^{* * *}$ & $<0.001$ \\
\hline AChE activity (nmol/mg protein) & $13.09 \pm 0.76$ & $12.19 \pm 0.58$ & 0.347 \\
\hline MAO activity ( $\mu \mathrm{mol} / \mathrm{h} / \mathrm{mg}$ protein $)$ & $0.31 \pm 0.02$ & $0.29 \pm 0.02$ & 0.61 \\
\hline $\mathrm{MAO}_{\mathrm{A}}$ activity $(\mu \mathrm{mol} / \mathrm{h} / \mathrm{mg}$ protein $)$ & $0.16 \pm 0.01$ & $0.15 \pm 0.01$ & 0.705 \\
\hline $\mathrm{MAO}_{\mathrm{B}}$ activity $(\mu \mathrm{mol} / \mathrm{h} / \mathrm{mg}$ protein $)$ & $0.13 \pm 0.01$ & $0.13 \pm 0.01$ & 1 \\
\hline
\end{tabular}

the change of another parameter such as stroke volume, which also exerts an influence on blood pressure. Therefore, it is less likely to contribute a role on the improvement of working memory. In addition, the increased respiratory rate is also observed but still in the normal range. However, a recent study reveals that the increase in respiration can also pack more oxygen into hemoglobin, which in turn can enhance brain oxygen supply and brain function [23]. Due to this line of evidence, we also suggest that the increase in brain oxygen supply induced by the elevation of respiration may also play a role on the memory enhancement. A decrease in diastolic pressure is also observed in subjects, 
TABLE 5: The effect of mulberry milk 2 servings/day on cortisol level and the activities of AChE, $\mathrm{MAO}, \mathrm{MAO}_{\mathrm{A}}$, and $\mathrm{MAO}_{\mathrm{B}}$ in saliva $(n=150$ /group) ${ }^{*} p$ value $<0.05$ and ${ }^{* * *} p$ value $<0.001$, respectively, compared to baseline.

\begin{tabular}{lccc}
\hline Parameter & \multicolumn{2}{c}{ Mulberry milk 2 servings/day } & \\
& Baseline & 6 weeks & \\
\hline Cortisol level $(\mathrm{ng} / \mathrm{ml})$ & $8.20 \pm 0.13$ & $6.87 \pm 0.09^{* * *}$ & $<.001$ \\
$\mathrm{AChE}$ activity $(\mathrm{nmol} / \mathrm{mg}$ protein) & $13.55 \pm 0.52$ & $12.02 \pm 0.56^{*}$ & 0.047 \\
$\mathrm{MAO}$ activity $(\mu \mathrm{mol} / \mathrm{h} / \mathrm{mg}$ protein) & $0.32 \pm 0.01$ & $0.27 \pm 0.01^{*}$ & 0.013 \\
$\mathrm{MAO}_{\mathrm{A}}$ activity $(\mu \mathrm{mol} / \mathrm{h} / \mathrm{mg}$ protein) & $0.16 \pm 0.01$ & $0.14 \pm 0.01^{*}$ & 0.034 \\
$\mathrm{MAO}_{\mathrm{B}}$ activity $(\mu \mathrm{mol} / \mathrm{h} / \mathrm{mg}$ protein) & $0.15 \pm 0.01$ & $0.13 \pm 0.01$ & 0.126 \\
\hline
\end{tabular}

who consumed an anthocyanin-rich mulberry milk 1 serving daily. Since the most important factor that determines diastolic blood pressure is total peripheral resistance, we suggest that a reduction of diastolic blood pressure may possibly associate with the reduction of total peripheral resistance due to the reduction of arterial contraction induced by the reduction of sensitivity to vasoactive agents such as norepinephrine via the permissive action of cortisol [24]. This study failed to show the dose-dependent manner because the tested product contains many ingredients so the effect of active ingredient could be masked by the other ingredients.

\section{Conclusion}

This study demonstrates the memory enhancing effect of an anthocyanin-rich mulberry milk in working-age adults. The possible underlying mechanisms may occur primarily via the suppression of cortisol. The high dose can also exert the positive modulation effect via the suppression effect on AChE, $\mathrm{MAO}$, and MAO-A. However, the increase brain oxygenation due to the increased respiration may also play a role and this point still requires further study to confirm it. The current data suggest that an anthocyanin-rich mulberry milk shows the potential to serve as the functional drink targeting at memory enhancement. Due to the suppression effect of cortisol, it may possibly exert the positive modulation effect on other stressrelated disorders such as anxiety, depression, and insomnia but these points also require further investigation.

\section{Data Availability}

I confirm that data are available and will be provided on request.

\section{Conflicts of Interest}

The authors declare that they have no conflicts of interest.

\section{Acknowledgments}

This study was supported by Integrative complementary Alternative Medicine Research Center in Research Institute for Human High Performance and Health Promotion, Khon Kaen University, Khon Kaen, Thailand, 40002. The authors would like to express the sincere gratitude to Mark One Innovation for supporting the tested products. In addition, we also would like to express our sincere gratitude to $\mathrm{Mr}$. Rit and Mrs. Yupin Thirakomen together with Dr. Petoon Maneepairoj from MK restaurant group for their supports.

\section{References}

[1] P. Salameh, A. Hajj, D. A. Badro, C. Abou Selwan, R. Aoun, and H. Sacre, "Mental health outcomes of the COVID-19 pandemic and a collapsing economy: perspectives from a developing country," Psychiatry Research, vol. 294, p. 113520, 2020.

[2] E. V. Goldfarb, "Participant stress in the COVID-19 era and beyond," Nature Reviews Neuroscience, vol. 21, no. 12, pp. 663-664, 2020.

[3] G. M. Peavy, D. P. Salmon, M. W. Jacobson et al., "Effects of chronic stress on memory decline in cognitively normal and mildly impaired older adults," The American Journal of Psychiatry, vol. 155, no. 12, pp. 1384-1391, 2009.

[4] J. Wattanathorn, N. Palachai, W. Thukham-mee, and S. Muchimapura, "Memory-Enhancing Effect of a Phytosome Containing the Combined Extract of Mulberry Fruit and Ginger in an Animal Model of Ischemic Stroke with Metabolic Syndrome," Oxidative Medicine and Cellular Longevity, vol. 2020, 9 pages, 2020.

[5] S. Kawvised, J. Wattanathorn, and W. Thukham-mee, "Neuroprotective and cognitive-enhancing effects of microencapsulation of mulberry fruit extract in animal model of menopausal women with metabolic syndrome," Oxidative Medicine and Cellular Longevity, vol. 2017, 13 pages, 2017.

[6] P. Kaewkaen, T. Tong-Un, J. Wattanathorn, S. Muchimapura, W. Kaewrueng, and S. Wongcharoenwanakit, "Mulberry fruit extract protects against memory impairment and hippocampal damage in animal model of vascular dementia," Evidence Based Complementary Alternative Medicine, vol. 2012, pp. 19, 2012.

[7] P. Kaewkaen, T. Tong-un, J. Wattanathorn, S. Muchimapura, W. Kaewrueng, and S. Wongcharoenwanakit, "Mulberry fruit mitigates alcohol neurotoxicity and memory impairment induced by chronic alcohol intake," American Journal of Applied Sciences, vol. 9, no. 4, pp. 484-491, 2012.

[8] W. Thukham-mee, J. Wattanathorn, W. Kirisattayakul, and P. Wannanon, "Effect of single administration of mulberry milk on the cognitive function of 6-12-year-old children: results from a randomized, placebo-controlled, crossover study," Oxidative Medicine and Cellular Longevity, vol. 2020, 10 pages, 2020.

[9] J. Wattanathorn, L. Mator, S. Muchimapura et al., "Positive modulation of cognition and mood in the healthy elderly volunteer following the administration of _Centella asiatica_," 
Journal of Ethnopharmacology, vol. 116, no. 2, pp. 325-332, 2008.

[10] N. Saenghong, J. Wattanathorn, S. Muchimapura et al., "Zingiber officinale improves cognitive function of the middle-aged healthy women," Evidence-Based Complementary and Alternative Medicine, vol. 2012, 9 pages, 2012.

[11] T. Peth-Nui, J. Wattanathorn, S. Muchimapura et al., "Effects of 12-week Bacopa monnieri consumption on attention, cognitive processing, working memory, and functions of both cholinergic and monoaminergic systems in healthy elderly volunteers," Evidence-Based Complementary and Alternative Medicine, vol. 2012, 2012.

[12] R. Sayer, E. Law, P. J. Connelly, and K. C. Breen, "Association of a salivary acetylcholinesterase with Alzheimer's disease and response to cholinesterase inhibitors," Clinical Biochemistry, vol. 37, no. 2, pp. 98-104, 2004.

[13] F. Doyle, P. E. Hucklebridge, and A. Clow, "Salivary monoamine oxidase A and B inhibitory activities correlate with stress," Life Sciences, vol. 59, no. 16, pp. 1357-1362, 1996.

[14] D. F. Holt, G. B. B. Sharman, and M. M. Palcic, "A continuous spectrophotometric assay for monoamine oxidase and related enzymes in tissue homogenates," Analytical Biochemistry, vol. 244, no. 2, pp. 384-392, 1997.

[15] K. A. Wesnes and J. Reynolds, "The effects on the cognitive function of healthy volunteers of a combination of acetyl-Lcarnitine, vinpocetine and huperzine A administered over 28 days," International Journal of Neurology and Neurotherapy, vol. 6, 2019.

[16] J. Fossella, T. Sommer, J. Fan et al., "Assessing the molecular genetics of attention networks," BMC Neuroscience, vol. 3, no. 1, p. 14, 2002.

[17] S. Enge, M. Fleischhauer, K.-P. Lesch, A. Reif, and A. Strobel, "Serotonergic modulation in executive functioning: linking genetic variations to working memory performance," Neuropsychologia, vol. 49, no. 13, pp. 3776-3785, 2011.

[18] C. Frydman, C. Camerer, P. Bossaerts, and A. Rangel, "MAOA-L carriers are better at making optimal financial decisions under risk," Proceedings of the Royal Society B: Biological Sciences, vol. 278, no. 1714, pp. 2053-2059, 2011.

[19] T. Steckler, G. Rammes, M. Sauvage et al., "Effects of the monoamine oxidase A inhibitor moclobemide on hippocampal plasticity in GR-impaired transgenic mice," Journal of Psychiatric Research, vol. 35, no. 1, pp. 29-42, 2001.

[20] Z. Cai, "Monoamine oxidase inhibitors: promising therapeutic agents for Alzheimer's disease (review)," Molecular Medicine Reports, vol. 9, no. 5, pp. 1533-1541, 2014.

[21] M. Maeda-Yamamoto, T. Saito, A. Nesumi et al., "Chemical analysis and acetylcholinesterase inhibitory effect of anthocyanin- rich red leaf tea (cv. Sunrouge)," Journal of the Science of Food and Agriculture, vol. 92, no. 11, pp. 2379-2386, 2012.

[22] A. Dreiseitel, G. Korte, P. Schreier et al., "Berry anthocyanins and their aglycons inhibit monoamine oxidases A and B," Pharmacological Research, vol. 59, no. 5, pp. 306-311, 2009.

[23] Q. Zhang, M. Roche, K. W. Gheres et al., "Cerebral oxygenation during locomotion is modulated by respiration," Nature Communications, vol. 10, no. 1, p. 5515, 2019.

[24] S. Yang and L. Zhang, "Glucocorticoids and vascular reactivity," Current Vascular Pharmacology, vol. 2, no. 1, pp. 1-12, 2004. 